\title{
BMJ Open Economic evaluation of aerobic exercise training in older adults with vascular cognitive impairment: PROMoTE trial
}

\author{
Jennifer C Davis, ${ }^{1,2}$ Ging-Yuek Robin Hsiung, ${ }^{3}$ Stirling Bryan, ${ }^{4}$ John R Best, ${ }^{1,2}$ \\ Janice J Eng, ${ }^{1}$ Michelle Munkacsy, ${ }^{1,2}$ Winnie Cheung, ${ }^{1,2}$ Bryan Chiu, ${ }^{1,2}$ \\ Claudia Jacova, ${ }^{3}$ Philip Lee, ${ }^{5}$ Teresa Liu-Ambrose ${ }^{1,2}$
}

To cite: Davis JC, Hsiung GYR, Bryan S, et al. Economic evaluation of aerobic exercise training in older adults with vascular cognitive impairment: PROMoTE trial. BMJ Open 2017;7:e014387. doi:10.1136/bmjopen-2016014387

- Prepublication history for this paper is available online. To view these files please visit the journal online (http://dx.doi.org/10.1136/ bmjopen-2016-014387).

Received 21 September 2016 Revised 9 February 2017 Accepted 24 February 2017

CrossMark

For numbered affiliations see end of article.

Correspondence to Dr Jennifer C Davis; Jennifer. davis@ubc.ca

\section{ABSTRACT}

Background/objectives: Evidence suggests that aerobic exercise may slow the progression of subcortical ischaemic vascular cognitive impairment (SIVCI) by modifying cardiovascular risk factors. Yet the economic consequences relating to aerobic training (AT) remain unknown. Therefore, our primary objective was to estimate the incremental cost per qualityadjusted life years (QALYs) gained of a thrice weekly AT intervention compared with usual care.

Design: Cost-utility analysis alongside a randomised trial.

Setting: Vancouver, British Columbia, Canada.

Participants: 70 adults (mean age of 74 years, $51 \%$ women) who meet the diagnostic criteria for mild SIVCI. Intervention: A 6-month, thrice weekly, progressive aerobic exercise training programme compared with usual care (CON; comparator) with a follow-up assessment 6 months after formal cessation of aerobic exercise training.

Measurements: Healthcare resource usage was estimated over the 6-month intervention and 6-month follow-up period. Health status (using the EQ-5D-3L) at baseline and trial completion and 6-month follow-up was used to calculate QALYs. The incremental costutility ratio (cost per QALY gained) was calculated.

Results: QALYs were both modestly greater, indicating a health gain. Total healthcare costs (ie, 1791 1369 $\{2015 \$ C A D\}$ at 6 months) were greater, indicating a greater cost for the thrice weekly AT group compared with CON. From the Canadian healthcare system perspective, the incremental cost-utility ratios for thrice weekly AT were cost-effective compared with CON, when using a willingness to pay threshold of $\$ C A D$ 20000 per QALY gained or higher.

Conclusions: AT represents an attractive and potentially cost-effective strategy for older adults with mild SIVCI.

Trial registration number: NCT01027858.

\section{INTRODUCTION}

Cerebrovascular disease is the second most common aetiology contributing to dementia in older adults ${ }^{-4}$ and may be the most underdiagnosed and yet most treatable form
Strengths and limitations of this study

- Our study is one of the first to investigate together the economic and health consequences relating to aerobic training.

- Very few randomised controlled trials with concurrent economic evaluations of exercise have been conducted in populations at risk for dementia such as those with vascular cognitive impairment.

- There was wide variability in the cost estimates and outliers due to a smaller sample size.

of cognitive dysfunction in older adults. ${ }^{5}$ Vascular cognitive impairment (VCI) defined as the loss of cognitive function due to vascular burden in the brain-is a prevalent condition that places a growing burden on the healthcare system. ${ }^{6}$ Cerebral small vessel disease plays a critical role in covert ischaemia and the development of subcortical ischaemic vascular cognitive impairment (SIVCI), ${ }^{7}$ the most common form of VCI_. ${ }^{8}$ SIVCI is defined by the presence of white matter lesions (WMLs) and lacunar infarcts and has the clinical consequence of increased dementia risk. ${ }^{8}{ }^{9}$ Research has demonstrated that one-third of all dementias are attributable to VCI. ${ }^{10-12}$ More specifically, the proportion of vascular dementia attributable to small-vessel disease ranges from 36 to $67 \% .^{13} 14$ The worldwide economic burden of dementia is increasing at an unprecedented rate. In 2015 , a $35 \%$ increase led to a worldwide annual estimate of 818 billion US dollars. The worldwide costs of dementia are expected to exceed 1 trillion US dollars by 2018. ${ }^{15}$ Notably, vascular dementia has among the highest annual direct costs and highest hospitalisation-related costs compared with other dementias such as Alzheimer's disease. ${ }^{16}$ The average annual cost per patient with VCI was $\$ 33740,{ }^{6}$ compared with a variable range of $\$ 1500-\$ 91000$ 
for Alzheimer's disease. The costs per VCI admission were $\sim \$ 9545$ with the average number of admissions increasing through the progression of the disease. ${ }^{6}$

Epidemiological data suggest that modification of vascular risk factors may be beneficial in slowing the progression of VCI. ${ }^{17-20}$ Hence, aerobic-based exercise training is one promising approach to delay the progression of VCI by reducing key vascular risk factors associated with metabolic syndrome. What remains unknown is whether aerobic-based exercise training as an intervention strategy compared with 'usual care' for individuals with mild SIVCI is a cost-effective strategy. Until now, the simultaneous impact of healthcare costs and consequences remains unknown. It is an essential next step to provide an estimate of the costs and consequences (ie, health gains or losses) related to the aerobic training (AT) intervention, given that this type of intervention could be delivered at a population level and thus have an immense impact.

Therefore, we conducted a concurrent economic evaluation with individual level data on cost and effectiveness outcomes collected during a proof-of-concept single-blinded randomised controlled trial-the Promotion Of the Mind Through Exercise (PROMoTE) trial. ${ }^{21}$ Our primary objective was to determine the incremental cost-utility ratio (incremental cost per incremental quality-adjusted life year (QALY) gained) of thrice weekly AT compared with usual care among individuals with mild SIVCI.

\section{METHODS}

\section{Overview of economic evaluation}

This cost-utility analysis was conducted concurrently with a 6-month proof-of-concept single-blinded randomised controlled trial with a 6-month follow-up study (ie, 6 months postintervention). ${ }^{21}{ }^{22}$ The details of the PROMoTE trial were previously reported. ${ }^{21}{ }^{22}$ Measurements were made at three times: baseline, end of the intervention period (6 months postrandomisation) and 6-month postintervention (ie, 12 months postrandomisation). Of 440 individuals screened for eligibility, 70 were deemed eligible for this economic evaluation. This economic evaluation used a Canadian healthcare system perspective, and a 6-month (ie, trial completion) and a 12-month (ie, 6-month postintervention) time horizon for the primary economic evaluation assessing the efficiency of the thrice weekly progressive AT and usual care plus education compared with the usual care plus education (CON; comparator) group. Participants in the CON group received usual care as well as monthly educational materials about VCI and healthy diet. However, no specific information regarding physical activity was provided. Briefly, usual care included whatever healthcare services a patient with mild SIVCI would usually receive in their clinical care. The main outcome for the cost-utility analysis was the incremental cost per QALY gained. We obtained approval for this study from the University of British Columbia Clinical Ethics Review Board (H13-00715).

We previously described study design, participant recruitment, randomisation, demographics, methods and results of the PROMoTE trial. ${ }^{21}$ We recruited participants from the University of British Columbia Hospital Clinic for $\mathrm{AD}$ and related disorders, the Vancouver General Hospital Stroke Prevention Clinic and specialised geriatric clinics in Metro Vancouver, BC. Recruitment occurred between December 2009 and April 2014 with randomisation occurring on an ongoing basis. The assessors were blinded to the participants' group allocation. The primary outcome measures for the PROMoTE study were the Alzheimer's Disease Assessment Scale Cognitive Subscale (ADAS- $\operatorname{Cog}^{23}$ ), the Executive Interview (EXIT-25 ${ }^{24}$ ) and the Alzheimer's Disease Co-operative Study-Activities of Daily Living $\left(\right.$ ADCS-ADL $\left.{ }^{25}\right)$. Secondary outcome measures included executive functions, cardiovascular capacity, physical activity level, physiological markers and health-related quality of life. We included 70 community dwelling older adults who were diagnosed with SIVCI, ${ }^{26}$ which requires the presence of cognitive syndrome ${ }^{21}$ and small vessel ischaemic disease. ${ }^{21}$ Other inclusion criteria included: (1) Montreal Cognitive Assessment (MoCA) ${ }^{27}$ score $<26$ at screening; (2) Mini-Mental State Examination (MMSE) $^{28}$ score of $\geq 20$ at screening; (3) community-dwelling; (4) live in Metro Vancouver; (5) had a caregiver, family member or friend who interacted with him/her on a weekly basis; (6) sufficient ability to read, write and speak English; (7) acceptable visual and auditory acuity to complete psychometric tests; (8) stable on a fixed dose of cognitive medications that is not expected to change during the 6-month intervention period; (9) provided a personally signed and dated informed consent document indicating that the individual (or a legally acceptable representative) has been informed of all pertinent aspects of the trial; (10) able to walk independently and (11) in sufficient health to participate in the study's aerobic-based exercise training programme.

\section{Costs}

We tracked healthcare resource usage prospectively. Our primary method used cost diaries where participants were asked to fill out a monthly diary detailing any health resource usage. We also telephoned participants every 3 months using a health resource usage questionnaire. For individuals who did not fill out their calendars, the health resource usage questionnaire was the primary mode of healthcare resource usage data collection. For participants who missed the 3-month follow-up telephone call and who did not return their calendar, they were asked to recall their healthcare resource usage over the 6-month intervention period. We also collected healthcare resource usage for the 6-month follow-up period postintervention. We analyse these end points separately (ie, trial completion at 6 months and 
follow-up completion at 1 year). The healthcare resource usage questionnaire included the following categories: any visits to healthcare professionals (including general practitioners, specialists, physiotherapists, etc); all visits, admissions or procedures carried out in a hospital and laboratory and diagnostic tests. We calculated the costs of delivering the thrice weekly AT intervention and the CON group. Our base case analysis considered the costs of all healthcare resource use. Research protocol-driven costs were excluded from our analysis.

A unit cost was assigned for each component of healthcare resource usage. Costs for admission to hospital were based on the fully allocated cost model of a tertiary care hospital, Vancouver General Hospital. We based costs on fee for service rates from the British Columbia Medical Services Plan 2013 price list for all healthcare professional-related costs. Unit costs for specialised services (ie, physiotherapy, chiropractic or naturopathic medicine) were taken from the BC Association website for each specialty. We inflated costs to 2015 Canadian dollars using the consumer price index reported by Statistics Canada. Given that our analytic time horizon was $\leq 12$ months, discounting was not applied.

\section{Effectiveness outcome}

Briefly, we assessed health status using the EQ-5D-3L. ${ }^{29}$ The EQ-5D-3L is a short five-item multiple choice questionnaire that measures an individual's health-related quality of life (HRQoL) and health status according to the following five domains: mobility, self-care, usual activities, pain and anxiety/depression. ${ }^{29}$ Each domain has three possible options: no problems, some problems or severe problems. The EQ-5D-3L health state utility values (HSUVs) at each time point are bounded from -0.54 to 1.00 where a score of $<0$ is indicative of a health state worse than death. The HSUVs represent values that individuals within society assign-these are Canadian societal values for given health states. ${ }^{30}$

We administered the EQ-5D-3L at baseline, trial completion and at the 6-month follow-up period to patients and a patient proxy (ie, see below under 'Caregiver'). From these data points, we calculated the total QALYs lost or gained at 6 (trial completion) and 12 (follow-up completion) months for the two experimental groups. We used multiple linear regression to calculate the incremental QALYs based on patient and proxy ratings for each participant adjusted for the baseline utility score. Baseline utility scores are often imbalanced between treatment arms. Given that a patient's utility score at baseline is most often highly correlated with that individual's QALYs over the study period, failure to control for this imbalance can lead to a misleading ICER. As such, we followed the recommendations of Manca and Palmer $^{31}$ using multiple linear regression to control for imbalances in baseline utility scores between the two treatment groups. All statistical analyses were carried out using STATA V.10.0.
Caregiver (proxy)

The caregivers had to be able to read, write and speak English in which the questionnaires were provided with acceptable visual and auditory acuity. Caregivers completed the EQ-5D-3L from their own perspective of the participant (ie, proxy's own perspective).

\section{Adverse events and mortality}

Participants were advised to report any adverse effects due to the intervention. Our safety monitoring committee reviewed all adverse events on a monthly basis.

\section{Handling missing data}

In the PROMoTE study, $17 \%$ of participants had incomplete 6 -month health resource usage data and $7 \%$ had incomplete 6-month EQ-5D-3L data at trial completion including dropouts. For the 6-month follow-up period, $19 \%$ of participants had incomplete 6-month follow-up health resource usage data and 19\% had incomplete 6-month follow-up EQ-5D-3L data. The reasons for missing data included: drop out, participant burden and administration error. We calculated the cost and effectiveness estimates for available cases (dropping observations with missing values), complete case sets and an imputed data set.

We examined the pattern of missing data using the STATA code: 'mvpatterns'. Missing data appeared to be missing at random, and therefore, we imputed missing data using Bayesian analyses following recommendations, ${ }^{31-34} 35$ in which all baseline study variables (including treatment assignment) were used to create 40 imputed data sets; parameter estimates and SEs were pooled across the 40 data sets. For multiple imputation, we used the 'mi imput mvn' procedure in STATA. The imputed data are reported as our base case analysis. We report the results using deletion of missing data as our sensitivity analysis (ie, complete case analysis).

\section{Cost-utility analysis}

We calculated the incremental cost-utility ratio for thrice weekly AT compared with the CON group twice using the patient-rated EQ-5D-3L and the caregiver proxy-rated EQ-5D-3L. Briefly, the incremental cost-utility ratio provides an index of the cost per QALY gained at intervention completion (ie, 6 months) and cost per QALY gained at 6-month postintervention (ie, 12 months). The incremental cost-utility ratio is the ratio between the difference in total mean costs between the AT and the CON groups and the difference in the mean QALY gained between the AT and the CON groups.

$$
\text { ICER }=\frac{\text { Cost of AT }- \text { Cost of CON }}{\text { Effect of AT }- \text { Effect of CON }}
$$

Nested imputation and nonparametric bootstrapping were used to model uncertainty around the estimates for costs and effectiveness. For each of the 40 cycles, we imputed missing values and bootstrapped the complete 
data set. For each cycle of imputation and bootstrapping, we calculated the total healthcare resource use cost and QALYs according to group allocation. The results of each cycle of imputation for participants were averaged in each of the two participant groups. The contribution of each cost item in relation to the total healthcare resource use was estimated for each group. Plots of the costeffectiveness plane and cost-effectiveness acceptability curves were generated based on 5000 iterations of nested imputation/bootstrapping using Fiellers' method to generate $95 \%$ confidence ellipses for the joint distribution of cost and effectiveness outcomes. ${ }^{36}$

The differences in mean costs and health outcomes in each group were expressed by reporting the incremental cost per QALY (ie, the incremental cost-utility ratio). The observed health benefit (ie, QALY) difference was close to zero; therefore, we used 5000 bootstrapped replications of mean cost and QALY differences. ${ }^{37}$ We used these values to generate cost-utility acceptability curves to estimate the probability that thrice weekly AT is considered cost-effective compared with $\mathrm{CON}$ over a select range of willingness to pay values. ${ }^{38}$

\section{Sensitivity analysis}

For our sensitivity analysis, we restricted our data to a complete case analysis, thus including only participants for whom we had complete cost and effectiveness data. We applied multiple imputation, bootstrapped CI estimation, adjustment for imbalances in baseline utility and bootstrapped estimates of the incremental cost-utility ratios.

\section{RESULTS}

\section{Baseline characteristics and exercise compliance}

Seventy-one eligible participants were randomised to AT or CON. One participant was deemed ineligible due to the presence of mixed dementia detected after randomisation and was excluded from all analyses. As such, our analytic sample consisted of 70 participants. Table 1 provides the baseline descriptive characteristics separated by the study group. Average class attendance was $68 \%$ for the AT group.

\section{Healthcare use and costs}

Complete healthcare resource usage data were provided by $58(83 \%)$ participants at 6 months and $57(81 \%)$ participants at 12 months. Response rates for healthcare usage data were comparable across the two participant groups. Unit costs for healthcare cost items are provided in table 2. In summary (table 2), the mean (SD) costs (2015 \$CAD) for healthcare professional visits, admissions to hospital and laboratory tests/investigations at 6 months were as follows: 940 (1194), 187 (325) and 113 (128). The mean (SD) costs (2015 \$CAD) for healthcare professional visits, admissions to hospital and laboratory tests/investigations at 6 months were as follows: 682 (465), 554 (1648) and 108 (132). The mean total healthcare resource usage costs for the control group (2015\$CAD) at 6 and 12 months were 1434 (1674) and 2964 (2947). The mean total healthcare resource usage costs for the AT group (2015 \$CAD) at 6 and 12 months were as follows: 1434 (1674) and 2964 (2947).

\section{Health outcomes}

Complete data for the EQ-5D-3L at baseline were provided by $69(99 \%)$ patients and $63(90 \%)$ caregivers. Complete data for the EQ-5D-3L at 6 months were provided by $65(93 \%)$ patients and $54(77 \%)$ caregivers. Complete data for the EQ-5D-3L at 12 months were provided by $57(81 \%)$ patients and $49(70 \%)$ caregivers. The response rates of patients or caregivers for dropouts were comparable between treatment groups. The mean EQ-5D-3L at 6 and 12 months and adjusted incremental QALYs for patients and caregivers are provided in table 3.

\section{Adjusting QALYs for imbalances in baseline utility Imputed case analysis}

After controlling for imbalances in baseline utility, the mean (SD) incremental QALY after 6 months calculated using the EQ-5D-3L was $0.82(0.06)$ as rated by patients and $0.83(0.06)$ as rated by the caregivers' perspective for the patients in the AT group and $0.78(0.09)$ as rated by patients and $0.79(0.12)$ as rated by the caregivers' perspective for the patients in the CON group (table 3). After controlling for imbalances in baseline utility, the mean (SD) incremental QALY after 12 months calculated using the EQ-5D-3L was $0.82(0.06)$ as rated by patients and $0.83(0.05)$ as rated by the caregivers' perspective for the patients in the AT group and $0.78(0.08)$ as rated by patients and $0.79(0.10)$ as rated by the caregivers' perspective for the patients in the CON group (table 3).

\section{Complete cases analysis}

After controlling for imbalances in baseline utility, the mean (SD) incremental QALY after 6 months calculated using the EQ-5D-3L was $0.82(0.06)$ as rated by patients and $0.83(0.05)$ as rated by the caregivers' perspective for the patients in the AT group and $0.78(0.09)$ as rated by patients and $0.78(0.12)$ as rated by the caregivers' perspective for the patients in the CON group (table 3). After controlling for imbalances in baseline utility, the mean (SD) incremental QALY after 12 months calculated using the EQ-5D-3L was $0.82(0.03)$ as rated by patients and $0.82(0.01)$ as rated by the caregivers' perspective for the patients in the AT group and $0.78(0.05)$ as rated by patients and $0.78(0.03)$ as rated by the caregivers' perspective for the patients in the $\mathrm{CON}$ group.

\section{Cost-utility analysis}

From the Canadian healthcare system perspective, the incremental cost-utility ratios for thrice weekly AT were cost-effective compared with the comparator group, when using a willingness to pay threshold of \$CAD 20000 per QALY gained or higher. Specifically, on the point 
Table 1 Baseline characteristics of participants

\begin{tabular}{|c|c|c|}
\hline Variables at baseline & $\begin{array}{l}\text { CON group } \\
n=35 \\
\text { Mean (SD) or } \\
n(\%) \text { or } \\
\text { median (IQR) }\end{array}$ & $\begin{array}{l}\text { AT group } \\
\mathrm{n}=35 \\
\text { Mean (SD) or } \\
\mathrm{n}(\%) \text { or } \\
\text { median (IQR) }\end{array}$ \\
\hline \multicolumn{3}{|l|}{ Descriptive variables and covariates } \\
\hline Age, years & $73.7(8.3)$ & $74.8(8.4)$ \\
\hline Gender, female & $17(49 \%)$ & $19(54 \%)$ \\
\hline Education, >high school & 27 (82\%) & $24(69 \%)$ \\
\hline Functional Comorbidity Index & $2.8(2.2)$ & $2.8(1.5)$ \\
\hline Hypertensive, yes & $20(61 \%)$ & $17(49 \%)$ \\
\hline Mini-mental state examination & $26.4(3.1)$ & $26.3(2.7)$ \\
\hline Montreal cognitive assessment & $21.7(4.4)$ & 20.7 (3.3) \\
\hline Waist-to-hip ratio & $0.93(0.07)$ & $0.88(0.08)$ \\
\hline Short physical performance battery & $10.51(1.20)$ & $10.62(1.86)$ \\
\hline Time-up-and-go (s) & $8.67(2.26)$ & $8.82(2.36)$ \\
\hline Physiological profile assessment & $0.94(1.42)$ & $0.94(1.39)$ \\
\hline \multicolumn{3}{|l|}{ Medications } \\
\hline Taking beta blockers, yes & $7(20 \%)$ & $7(20 \%)$ \\
\hline Central-effecting medications, no & $0.5(1.0)$ & $0.6(0.9)$ \\
\hline Total medications, no & $4.2(3.4)$ & $3.5(2.7)$ \\
\hline \multicolumn{3}{|l|}{ Primary clinical and economic outcome variables } \\
\hline Alzheimer's disease assessment scale, cognition & $10.2(5.4)$ & $11.7(5.5)$ \\
\hline Executive interview & $13.3(6.4)$ & $13.7(4.7)$ \\
\hline ADCS-ADL & $46.5(5.1)$ & $46.1(6.8)$ \\
\hline \multirow[t]{2}{*}{ EQ-5D-3L (patient rated) } & $0.797(0.109)$ & $0.822(0.072)$ \\
\hline & $0.817(0.135)$ & $0.826(0.108)$ \\
\hline \multirow[t]{2}{*}{ EQ-5D-3L (caregiver rated) } & $0.799(0.136)$ & $0.829(0.064)$ \\
\hline & $0.826(0.117)$ & $0.843(0.108)$ \\
\hline \multicolumn{3}{|l|}{ Secondary outcome variables } \\
\hline Stroop Test 3-2 (s) & $57.12(24.13)$ & $67.82(28.36)$ \\
\hline Trail making test B-A (s) & $75.18(83.27)$ & $59.70(42.28)$ \\
\hline Digit span forward-backward & $3.8(1.95)$ & $3.37(2.44)$ \\
\hline 6-minute walk (m) & $486.9(97.9)$ & $502.8(98.4)$ \\
\hline Weight $(\mathrm{kg})$ & $72.39(14.11)$ & $70.05(14.31)$ \\
\hline Body mass index & $26.54(3.97)$ & $25.26(3.54)$ \\
\hline Resting heart rate (bpm) & $70.24(15.10)$ & $67.26(12.38)$ \\
\hline Resting systolic blood pressure $(\mathrm{mm} \mathrm{Hg})$ & $132.29(18.66)$ & $139.80(17.73)$ \\
\hline Resting diastolic blood pressure (mm $\mathrm{Hg})$ & $76.71(11.38)$ & $80.26(10.05)$ \\
\hline Physical activity scale for the elderly & $118.59(55.41)$ & $124.44(73.47)$ \\
\hline
\end{tabular}

estimates from our base case analysis, we found that AT is more effective and also more costly than the CON alternative. Figure 1A (using the patient's own ratings of their health status) demonstrates that for three times weekly $\mathrm{AT}$ at 6 months (ie, intervention completion) compared with $\mathrm{CON}$, most of the bootstrapped cycles $(>80 \%$ of the 4000 cycles) were represented in the northeast quadrant. Figure 1B (using the caregiver's ratings of the patient's health status) demonstrates that for three times weekly $\mathrm{AT}$ at 6 months (ie, intervention completion) compared with CON, most of the bootstrapped cycles $(>80 \%$ of the 4000 cycles) were represented in the northeast quadrant. Figure $1 \mathrm{C}, \mathrm{D}$ (using the patient's own ratings and caregiver's ratings of their health status, respectively) demonstrates that for three times weekly AT at 12 months (ie, intervention completion) compared with $\mathrm{CON}$, most of the 4000 bootstrapped cycles were represented in the northeast quadrant. Figure 2A-D reports the costeffectiveness acceptability curves highlighting the probability of the AT being cost-effective over different willingness to pay values.

\section{Sensitivity analysis}

Our complete case analysis demonstrated the same trend with regard to a significant improvement in QALYs and an overall increase in health resource usage costs for the AT group.

\section{DISCUSSION}

Among a population of individuals at high risk for future cognitive decline, this study demonstrated, using a 
Table 2 Unit costs for each component of resource usage

\begin{tabular}{|c|c|c|c|c|}
\hline Item & $\begin{array}{l}\text { 6-month HRU } \\
2015 \text { CAN\$ } \\
\text { Mean (SD) } \\
\text { Median (IQR) } \\
\end{array}$ & $\begin{array}{l}\text { 12-month HRU } \\
2015 \text { CAN\$ } \\
\text { Mean (SD) } \\
\text { Median (IQR) } \\
\end{array}$ & Unit & Reference \\
\hline $\begin{array}{l}\text { Cost of delivering control } \\
\text { group }\end{array}$ & 0 & - & $\begin{array}{l}\text { Cost per person } \\
\text { year }\end{array}$ & Study records \\
\hline $\begin{array}{l}\text { Cost of delivering thrice } \\
\text { weekly aerobic training }\end{array}$ & 576 & - & $\begin{array}{l}\text { Cost per person } \\
\text { year }\end{array}$ & Study records \\
\hline Healthcare professional visit, & $940(1194)$ & $\begin{array}{l}682(465) \\
632(726)\end{array}$ & Cost per person & 2013 Medical services plan \\
\hline $\begin{array}{l}\text { mean (SD) } \\
\text { Admission to hospital }\end{array}$ & $\begin{array}{c}586(1097) \\
187(325) \\
0(277)\end{array}$ & $\begin{array}{c}632(726) \\
552(1648) \\
0(207)\end{array}$ & Cost per person & $\begin{array}{l}2005 \text { Vancouver General Hospital } \\
\text { fully allocated cost model }{ }^{*}\end{array}$ \\
\hline $\begin{array}{l}\text { Emergency department } \\
\text { presentations }\end{array}$ & 42 & & Cost per hour & $\begin{array}{l}2005 \text { Vancouver General Hospital } \\
\text { fully allocated cost model }{ }^{*}\end{array}$ \\
\hline $\begin{array}{l}\text { Laboratory procedures, mean } \\
\text { (SD) }\end{array}$ & $\begin{array}{r}113(128) \\
44(204)\end{array}$ & $\begin{array}{r}108(132) \\
59(129)\end{array}$ & Cost per person & 2009 Medical services plan \\
\hline
\end{tabular}

Canadian healthcare system perspective, that the incremental cost per QALY gained by participating in thrice weekly AT was more effective and more costly than the usual care plus education group. We observed a trend towards improvement in the adjusted incremental QALYs determined from the EQ-5D-3L (by patients and proxies) for the AT group compared with the usual care plus education group at trial completion and 6-month follow-up. Importantly, AT is an alternative to resistance training and is accessible to older adults with mild SIVCI. Further, the delivery of a walking programme on an individual basis requires a low financial investment (ie, the cost of walking poles) by an individual. As such, the results of this economic evaluation represent a substantive contribution to the evidence base on how to efficiently minimise cognitive decline among those with mild SIVCI.

The findings of this study build on previous research demonstrated that AT has significant and beneficial effects on overall health-related quality of life and quality of life more broadly. ${ }^{39}$ The overall incremental cost-utility ratios were not significantly different regardless of whether QALYs were ascertained from patientreported or proxy-reported health status using the EQ-5D-3L suggesting that for this population, use of patient or proxy ratings should not alter healthcare decision-making. The cost-effectiveness acceptability curves confirm that AT is the preferred treatment option for a wide range of plausible willingness to pay thresholds.

From both our sensitivity analyses, we found that all analyses supported the conclusions that AT resulted in clinically important gains in QALYs. However, our imputed case analysis demonstrated that the intervention was not cost-saving, while the complete case analysis demonstrated that the intervention was cost-saving. One potential explanation for this was that the complete case analysis may better reflect the per protocol findings (ie, those that had greater adherence to the trial).

Table 3 Results of imputed case analysis

\begin{tabular}{|c|c|c|c|c|}
\hline & $\begin{array}{l}\text { CON at } \\
6 \text { months } \\
\text { Mean (SD) }\end{array}$ & $\begin{array}{l}\text { CON at } \\
12 \text { months } \\
\text { Mean (SD) }\end{array}$ & $\begin{array}{l}\text { AT at } \\
6 \text { months } \\
\text { Mean (SD) }\end{array}$ & $\begin{array}{l}\text { AT at } \\
12 \text { months } \\
\text { Mean (SD) }\end{array}$ \\
\hline Cost of delivering programme per person (2015 CAN\$) & 0 (usual care) & 0 (usual care) & 730 & 730 \\
\hline Mean healthcare resource use cost (2015 CAN\$) per person & $1434(1674)$ & $2964(2947)$ & $956(861)$ & $2110(1857)$ \\
\hline \multicolumn{5}{|l|}{ Adjusted incremental QALY based on } \\
\hline EQ-5D-3L patient* & 0 (reference) & 0 (reference) & $0.804(0.080)$ & $0.800(0.075)$ \\
\hline EQ-5D-3L caregiver* & 0 (reference) & 0 (reference) & $0.806(0.096)$ & $0.810(0.078)$ \\
\hline \multicolumn{5}{|l|}{ Incremental cost (2015 \$CAD) } \\
\hline EQ-5D-3L patient & Reference & Reference & $1770(1369)$ & 3112 (2499) \\
\hline EQ-5D-3L caregiver & Reference & Reference & $1770(1369)$ & 3112 (2499) \\
\hline \multicolumn{5}{|l|}{ Incremental cost (2015 \$CAD) per QALY based on $\dagger$} \\
\hline EQ-5D-3L patient & Reference & Reference & 2129 & 3761 \\
\hline EQ-5D-3L caregiver & Reference & Reference & 2124 & 3715 \\
\hline
\end{tabular}

*Incremental QALYs are adjusted for the baseline utility using a linear regression model.

†ICER based on total HRU costs, fall related costs and cost of delivering programmes. 

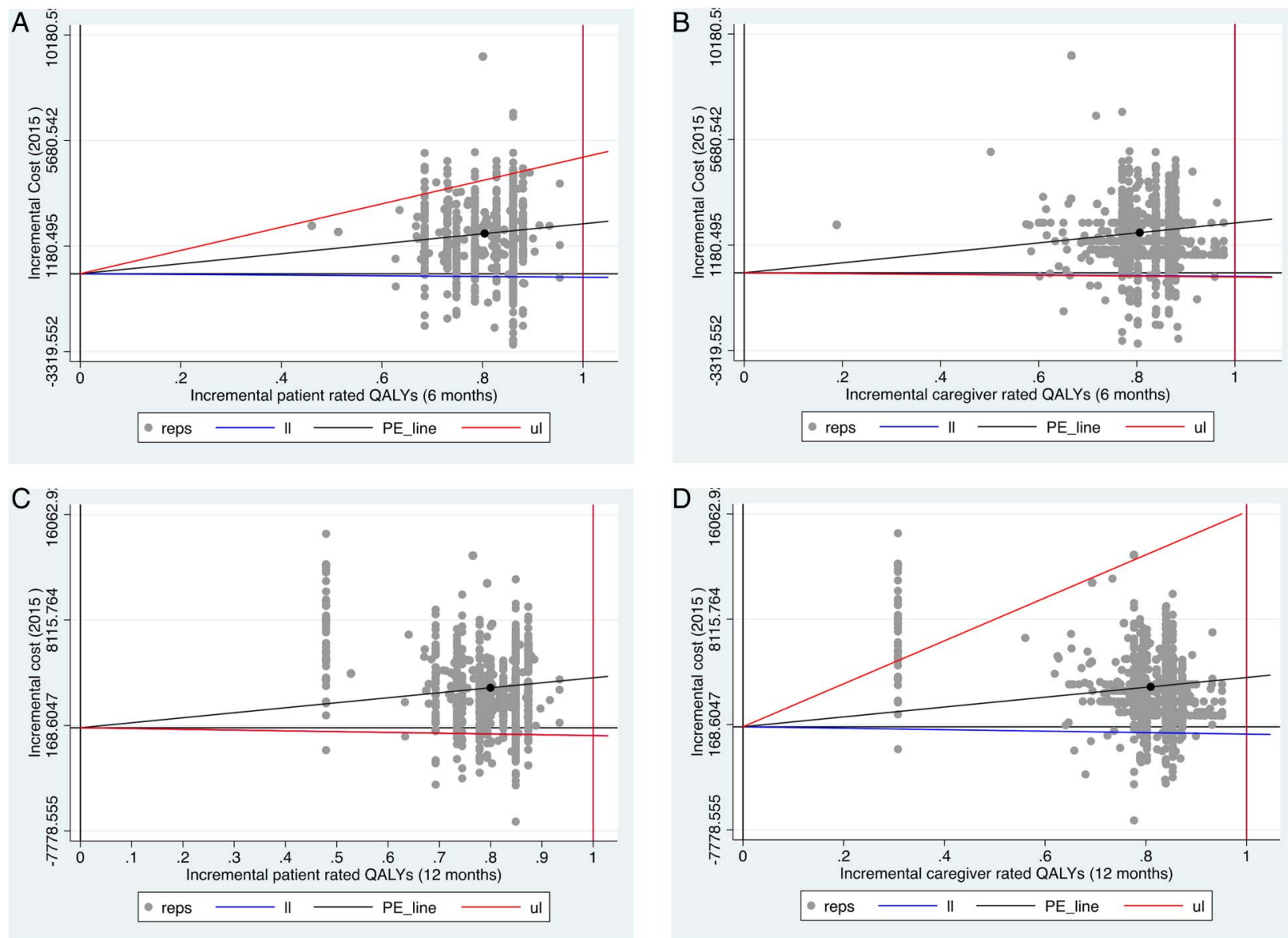

Figure 1 (A) Cost-effective plane (time horizon-6 months) depicting the 95\% confidence ellipses of incremental cost and effectiveness (patient-rated health status) for comparison between thrice weekly aerobic training and usual care (control, comparator). (B) Cost-effective plane (time horizon-6 months) depicting the $95 \%$ confidence ellipses of incremental cost and effectiveness (caregiver (patient-proxy) rated health status) for comparison between thrice weekly aerobic training and usual care (control, comparator). (C) Cost-effective plane (time horizon-12 months) depicting the 95\% confidence ellipses of incremental cost and effectiveness (patient-rated health status) for comparison between thrice weekly aerobic training and usual care (control, comparator). (D) Cost-effective plane (time horizon-12 months) depicting the $95 \%$ confidence ellipses of incremental cost and effectiveness (caregiver (patient-proxy) rated health status) for comparison between thrice weekly aerobic training and usual care (control, comparator).

The time horizon of our study was limited to the duration of the intervention (ie, 6 months) and the follow-up period (ie, 12 months). The number of randomised controlled trials of exercise conducted in populations at high risk for dementia is accumulating. ${ }^{40}$ One study demonstrated that resistance training post a 6-month (frequency of two to three times weekly) intervention significantly improved global cognitive function while maintaining executive and global benefits for at least 18 months postintervention. ${ }^{41} 42$ However, the number of randomised controlled trials of exercise among individuals diagnosed with SIVCI remains low. ${ }^{22}$ Given that cardiovascular risk factors play a primary role in the onset and progression of VCI, examining the cost-effectiveness of AT is a logical starting place. In adults with mild VCI, 6 months of thrice weekly progressive AT improved cognitive function, relative to CON. ${ }^{22}$ Previous research that AT in older adults has longer term health benefits that we hypothesise would be applicable to adults with mild SIVCI benefit of the intervention may be ideally captured by a longer time horizon. ${ }^{43}$ Further, the sample size of our study was small. As such, there was wide variability in the cost estimates and outliers (ie, \pm 3 SDs from the mean) had a stronger impact than would be expected in a larger sample. However, we did not have any reason to remove any health resource usage outliers in our intention to treat analysis. The health resource usage questionnaire may be subject to recall bias, thus causing potential underestimation of costs. To minimise recall bias, participants were provided with a monthly diary to track and report their healthcare resource usage. Given that cost underestimation may have occurred in both groups, we do not estimate any impact on the incremental cost-utility ratio given that this was a randomised controlled trial.

A key strength of our study is that it deals with a largely understudied yet important population. Importantly, this population actually may represent an ideal target population for intervention given that individuals have not yet 

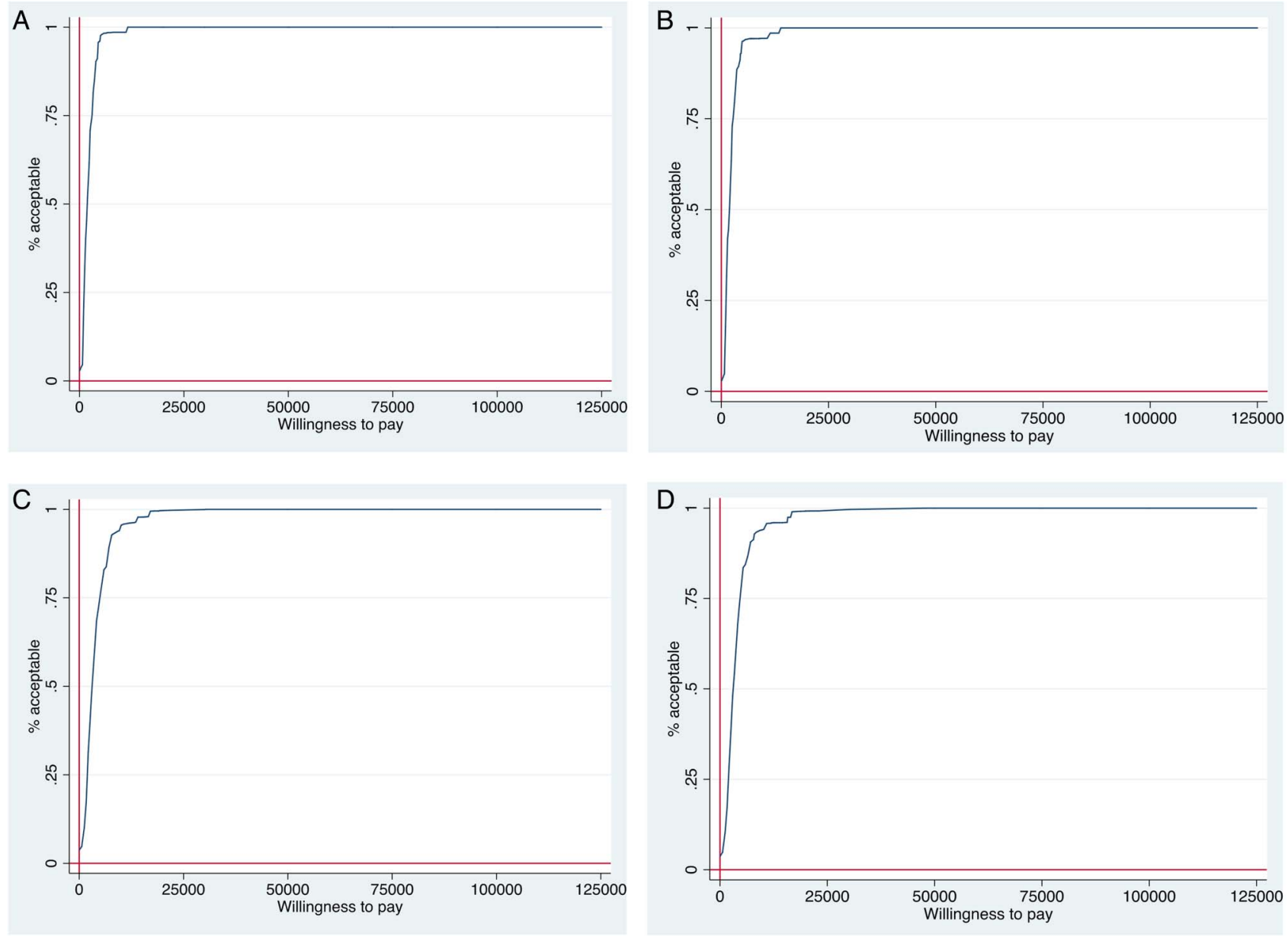

Figure 2 (A) Cost-effectiveness acceptability curve showing the probability that thrice aerobic training intervention is cost-effective compared to usual care over a range of values for the maximum acceptable ceiling ratio ( $\lambda$-willingness to pay) in the PROMoTE trial (6-month time horizon, patient-rated health status). (B) Cost-effectiveness acceptability curve showing the probability that thrice aerobic training intervention is cost-effective compared to usual care over a range of values for the maximum acceptable ceiling ratio ( $\lambda$-willingness to pay) in the PROMoTE trial (6-month time horizon, caregiver (patient-proxy)-rated health status). (C) Cost-effectiveness acceptability curve showing the probability that thrice aerobic training intervention is cost-effective compared to usual care over a range of values for the maximum acceptable ceiling ratio ( $\lambda$-willingness to pay) in the PROMoTE trial (12-month time horizon, patient-rated health status). (D) Cost-effectiveness acceptability curve showing the probability that thrice aerobic training intervention is cost-effective compared to usual care over a range of values for the maximum acceptable ceiling ratio ( $\lambda$-willingness to pay) in the PROMoTE trial (12-month time horizon, caregiver (patient-proxy) rated health status).

crossed the dementia threshold. Hence, it is important to gain a better understanding of the effectiveness and efficiency of targeted interventions. Given that even mildly impaired cognition may impede an individual's ability to self-assess their HRQoL we also used a patientproxy (ie, caregiver) assessment of the patient's health status. ${ }^{44}$ In this study, we found that the use of the patient or the proxy did not significantly alter our findings. In all instances, we observed a significant increase in QALYs at 6 and 12 months regardless of the rater. This is a useful observation because it suggests that among individuals with VCI, the rater should not result in changes in healthcare decision-making. Finally, a highly relevant strength of this study is that the intervention is widely accessible and relatively easy to implement for any community dwelling older adult who is able to walk. The low cost required by an individual to start walking is also appealing from an implementation perspective.

Our proof-of-concept findings suggest that this exercise (ie, AT) therapy delivered over a span of 6 months holds promise for improving cognitive function and health-related quality of life in older adults with mild VCI. While our findings suggest that this intervention is not cost-saving, it appears to be cost-effective depending on a decision maker's willingness to pay.

\section{Author affiliations}

${ }^{1}$ Department of Physical Therapy, University of British Columbia, Vancouver, British Columbia, Canada

${ }^{2}$ Centre for Hip Health and Mobility, Vancouver Coastal Research Institute, Vancouver, British Columbia, Canada 
${ }^{3}$ Department of Medicine, Division of Neurology, University of British Columbia, Vancouver, British Columbia, Canada

${ }^{4}$ Centre for Clinical Epidemiology and Evaluation, University of British Columbia, Vancouver, British Columbia, Canada

${ }^{5}$ Department of Medicine, Division of Geriatric Medicine, University of British Columbia, Vancouver, British Columbia, Canada

\section{Acknowledgements The authors thank the PROMoTE study participants.}

Contributors TL-A and JCD had full access to all the data in the study, take responsibility for the integrity of the data and the accuracy of the data analysis and involved in study concept and design. TL-A, JCD, SB and JRB contributed to acquisition, analysis or interpretation of data. JCD, TL-A and SB drafted the manuscript. JCD, G-YRH, SB, JRB, JJE, MM, WC, BC, CJ, PL and TL-A contributed to critical revision of the manuscript for important intellectual content. JCD and JRB involved in statistical analysis. TL-A, CJ, G-YRH, JJE, PL and JCD obtained funding. WC, MM and BC provided administrative, technical, or material support. TL-A, JCD, MM and WC contributed to study supervision.

Funding This study is jointly funded by the Canadian Stroke Network and the Heart and Stroke Foundation of Canada (grant number: PG-09-0443). TL-A is a Canada Research Chair in Physical Activity, Mobility, and Cognitive Neuroscience, a Michael Smith Foundation for Health Research (MSFHR) Scholar, a Canadian Institutes of Health Research (CIHR) New Investigator and a Heart and Stroke Foundation of Canada's Henry JM Barnett's Scholarship recipient. These funding agencies did not play a role in study design.

\section{Competing interests None declared.}

Provenance and peer review Not commissioned; externally peer reviewed.

Data sharing statement Unpublished data are available on request. Please email Dr Teresa Liu-Ambrose: teresa.ambrose@ubc.ca.

Open Access This is an Open Access article distributed in accordance with the Creative Commons Attribution Non Commercial (CC BY-NC 4.0) license, which permits others to distribute, remix, adapt, build upon this work noncommercially, and license their derivative works on different terms, provided the original work is properly cited and the use is non-commercial. See: http:// creativecommons.org/licenses/by-nc/4.0/

\section{REFERENCES}

1. Rockwood K, Wentzel C, Hachinski V, et al. Prevalence and outcomes of vascular cognitive impairment. Vascular Cognitive Impairment Investigators of the Canadian Study of Health and Aging. Neurology 2000;54:447-51.

2. Desmond DW, Erkinjuntti T, Sano M, et al. The cognitive syndrome of vascular dementia: implications for clinical trials. Alzheimer Dis Assoc Disord 1999;13(Suppl 3):S21-9.

3. Erkinjuntti T, Bowler JV, DeCarli CS, et al. Imaging of static brain lesions in vascular dementia: implications for clinical trials. Alzheimer Dis Assoc Disord 1999;13(Suppl 3):S81-90.

4. Pantoni L, Leys D, Fazekas F, et al. Role of white matter lesions in cognitive impairment of vascular origin. Alzheimer Dis Assoc Disord 1999;13(Suppl 3):S49-54.

5. Román GC. Vascular dementia may be the most common form of dementia in the elderly. J Neurol Sci 2002;203-204:7-10.

6. Ramos-Estebanez C, Moral-Arce I, Rojo F, et al. Vascular cognitive impairment and dementia expenditures: 7-year inpatient cost description in community dwellers. Postgrad Med 2012;124: 91-100.

7. O'Brien JT, Erkinjuntti T, Reisberg B, et al. Vascular cognitive impairment. Lancet Neurol 2003;2:89-98.

8. Román GC, Erkinjuntti T, Wallin A, et al. Subcortical ischaemic vascular dementia. Lancet Neurol 2002;1:426-36.

9. Vermeer SE, Longstreth WT Jr, Koudstaal PJ. Silent brain infarcts: a systematic review. Lancet Neurol 2007;6:611-19.

10. de Bruijn RF, Bos MJ, Portegies ML, et al. The potential for prevention of dementia across two decades: the prospective, population-based Rotterdam Study. BMC Med 2015;13:132.

11. Barnes DE, Yaffe K. The projected effect of risk factor reduction on Alzheimer's disease prevalence. Lancet Neurol 2011;10: 819-28.
12. Norton S, Matthews FE, Barnes DE, et al. Potential for primary prevention of Alzheimer's disease: an analysis of population-based data. Lancet Neurol 2014;13:788-94.

13. Jokinen $H$, Kalska $H$, Mäntylä $R$, et al. Cognitive profile of subcortical ischaemic vascular disease. J Neurol Neurosurg Psychiatr 2006;77:28-33

14. Kramer JH, Reed BR, Mungas D, et al. Executive dysfunction in subcortical ischaemic vascular disease. J Neurol Neurosurg Psychiatr 2002;72:217-20.

15. Wimo A, Guerchet M, Ali GC, et al. The worldwide costs of dementia 2015 and comparisons with 2010. Alzheimers Dement 2017;13:1-7.

16. Rojas G, Bartoloni L, Dillon C, et al. Clinical and economic characteristics associated with direct costs of Alzheimer's, frontotemporal and vascular dementia in Argentina. Int Psychogeriat 2011;23:554-61.

17. Abbott RD, Donahue RP, MacMahon SW, et al. Diabetes and the risk of stroke. The Honolulu Heart Program. JAMA 1987;257:949-52.

18. Knopman D, Boland LL, Mosley T, et al. Cardiovascular risk factors and cognitive decline in middle-aged adults. Neurology 2001;56:42-8.

19. Anstey KJ, Lipnicki DM, Low LF. Cholesterol as a risk factor for dementia and cognitive decline: a systematic review of prospective studies with meta-analysis. Am J Geriatr Psychiatry 2008;16:343-54.

20. Tzourio C, Anderson C, Chapman N, et al. Effects of blood pressure lowering with perindopril and indapamide therapy on dementia and cognitive decline in patients with cerebrovascular disease. Arch Intern Med 2003;163:1069-75.

21. Liu-Ambrose T, Eng JJ, Boyd LA, et al. Promotion of the mind through exercise (PROMoTE): a proof-of-concept randomized controlled trial of aerobic exercise training in older adults with vascular cognitive impairment. BMC Neurol 2010;10:14.

22. Liu-Ambrose T, Best JR, Davis JC, et al. Aerobic exercise and vascular cognitive impairment: a randomized controlled trial. Neurology 2016;87:2082-90.

23. Rosen WG, Mohs RC, Davis KL. A new rating scale for Alzheimer's disease. Am J Psychiatry 1984;141:1356-64.

24. Royall DR, Mahurin RK, Gray KF. Bedside assessment of executive cognitive impairment: the executive interview. J Am Geriatr Soc 1992;40:1221-6.

25. Galasko D, Bennett D, Sano M, et al. An inventory to assess activities of daily living for clinical trials in Alzheimer's disease. The Alzheimer's disease Cooperative Study. Alzheimer Dis Assoc Disord 1997;11(Suppl 2):S33-9.

26. Erkinjuntti T, Inzitari D, Pantoni L, et al. Research criteria for subcortical vascular dementia in clinical trials. J Neural Transm Suppl 2000;59:23-30.

27. Nasreddine ZS, Phillips NA, Bédirian V, et al. The montreal cognitive assessment, MoCA: a brief screening tool for mild cognitive impairment. J Am Geriatr Soc 2005;53:695-9.

28. Folstein MF, Folstein SE, McHugh PR. "Mini-mental state". A practical method for grading the cognitive state of patients for the clinician. J Psychiatr Res 1975;12:189-98.

29. Dolan P. Modeling valuations for EuroQol health states. Med Care 1997;35:1095-108.

30. Bansback N, Tsuchiya A, Brazier J, et al. Canadian valuation of EQ-5D health states: preliminary value set and considerations for future valuation studies. PLOS ONE 2012;7:e31115.

31. Manca A, Palmer S. Handling missing data in patient-level cost-effectiveness analysis alongside randomised clinical trials. Appl Health Econ Health Policy 2005;4:65-75.

32. Briggs A, Clark T, Wolstenholme J, et al. Missing...presumed at random: cost-analysis of incomplete data. Health Econ 2003;12:377-92.

33. Oostenbrink JB, Al MJ. The analysis of incomplete cost data due to dropout. Health Econ 2005;14:763-76.

34. Oostenbrink JB, AI MJ, Rutten-van Molken MP. Methods to analyse cost data of patients who withdraw in a clinical trial setting. Pharmacoeconomics 2003;21:1103-12.

35. Schafer JL. Analysis of incomplete multivariate data. London, England: Chapman \& Hall, 1997.

36. Laska EM, Meisner M, Siegel C. Statistical inference for cost-effectiveness ratios. Health Econ 1997;6:229-42.

37. Briggs $\mathrm{AH}$, Gray AM. Handling uncertainty when performing economic evaluation of healthcare interventions. Health Technol Assess 1999;3:1-134.

38. Fenwick E, Claxton K, Sculpher M. Representing uncertainty: the role of cost-effectiveness acceptability curves. Health Econ 2001;10:779-87. 
39. Awick EA, Wójcicki TR, Olson EA, et al. Differential exercise effects on quality of life and health-related quality of life in older adults: a randomized controlled trial. Qual Life Res 2015;24: 455-62.

40. Lautenschlager NT, Cox KL, Flicker L, et al. Effect of physical activity on cognitive function in older adults at risk for Alzheimer disease: a randomized trial. JAMA 2008;300:1027-37.

41. Fiatarone Singh MA, Gates N, Saigal N, et al. The Study of Menta and Resistance Training (SMART) study-resistance training and/or cognitive training in mild cognitive impairment: a randomized, double-blind, double-sham controlled trial. J Am Med Dir Assoc 2014:15:873-80.
42. Lam LC, Chan WM, Kwok TC, et al. Effectiveness of Tai Chi in maintenance of cognitive and functional abilities in mild cognitive impairment: a randomised controlled trial. Hong Kong Med $\mathrm{J}$ 2014;20(3 Suppl 3):20-3.

43. McCartney N, Hicks AL, Martin J, et al. Long-term resistance training in the elderly: effects on dynamic strength, exercise capacity, muscle, and bone. J Gerontol A Biol Sci Med Sci 1995;50:B97-104.

44. Makai P, Beckebans F, van Exel J, et al. Quality of life of nursing home residents with dementia: validation of the German version of the ICECAP-O. PLOS ONE 2014;9:e92016.

45. Bryan S, Hardyman W, Bentham P, et al. Proxy completion of EQ-5D in patients with dementia. Qual Life Res 2005;14:107-18. 